\title{
Effect of Nanoconfinement on Polymer Dynamics: Surface Layers and Interphases
}

\author{
M. Krutyeva, ${ }^{1}$ A. Wischnewski, ${ }^{1}$ M. Monkenbusch, ${ }^{1}$ L. Willner, ${ }^{1}$ J. Maiz, ${ }^{2}$ C. Mijangos, ${ }^{2}$ A. Arbe,${ }^{3}$ J. Colmenero,,${ }^{3,4}$ \\ A. Radulescu, ${ }^{5}$ O. Holderer, ${ }^{5}$ M. Ohl, ${ }^{6}$ and D. Richter ${ }^{1}$ \\ ${ }^{1}$ Jülich Centre for Neutron Science (JCNS) and Institute for Complex Systems (ICS), Forschungszentrum Jülich GmbH, \\ 52428 Jülich, Germany \\ ${ }^{2}$ Instituto de Ciencia y Tecnología de Polímeros, CSIC, 28006 Madrid, Spain \\ ${ }^{3}$ Centro de Física de Materiales CSIC-UPV/EHU, 20018 San Sebastián, Spain \\ ${ }^{4}$ Materials Physics Center (MPC) and Donostia International Physics Center, 20018 San Sebastián, Spain \\ ${ }^{5}$ JCNS at Outstation Garching, 85748 Garching, Germany \\ ${ }^{6}$ JCNS at Outstation Oak Ridge, Oak Ridge, Tennessee 37831, USA
}

(Received 2 October 2012; published 5 March 2013)

\begin{abstract}
We present neutron spin echo experiments that address the much debated topic of dynamic phenomena in polymer melts that are induced by interacting with a confining surface. We find an anchored surface layer that internally is highly mobile and not glassy as heavily promoted in the literature. The polymer dynamics in confinement is, rather, determined by two phases, one fully equal to the bulk polymer and another that is partly anchored at the surface. By strong topological interaction, this phase confines further chains with no direct contact to the surface. These form the often invoked interphase, where the full chain relaxation is impeded through the interaction with the anchored chains.
\end{abstract}

DOI: 10.1103/PhysRevLett.110.108303

PACS numbers: 82.35.Lr, 29.30.Hs, 81.05.Qk, 83.80.Sg

The investigation of liquids under nanoconfinement has been a topic of intense scientific scrutiny for decades [1]. The issues are glass transition, crystallization, and phase separation under confinement [2,3]. Recently, this interest has been amplified by the rising of nanotechnology that aims to create new properties by modifying materials at the nanoscale. Polymers are of particular interest since they offer a large range of applications such as coatings, lubrication, nanocomposites, and in the field of biological macromolecules, biosensors [4].

Close to a confining surface, the conformations of a polymer are significantly restricted [5]. In addition, the interactions with the surface will strongly affect the dynamics. Related issues such as adsorption, friction, network formation, effects on the entanglement density, and polymer density changes under confinement have been studied [6-9]. The importance of these phenomena thereby depends on the type of polymer, the specificity of the interactions, and the topology of the confinement. In particular, experimental results have been interpreted in terms of the formation of a glassy polymer layer close to surfaces [7]. Furthermore, the existence of an interphase with properties between those of the glassy layer and the bulk has been hypothesized [10-12].

A large number of experimental studies have focused on nanoparticles dispersed in a polymer matrix. Whereas for noninteracting polymers significant effects only occur at high particle loadings, the addition of nanoparticles that interact with a polymer matrix induces dramatic property changes for the resulting polymer nanocomposite $[7,9,10,13,14]$. In particular, it has been reported that the interaction between $\mathrm{OH}$ groups on the surface of nanoparticles and locally polar poly(ethylene oxide) (PEO) or polydimethylsiloxane (PDMS) chains lead to the formation of a glassy polymer layer [7,10,13]. Theoretical work and computer simulations of chain adsorption as a function of adsorption strength reveal the existence of different chain conformations including trains, loops, and tails [14].

Here, we present an investigation on the dynamics of PDMS chains confined in anodic aluminum oxide (AAO) nanopores. We find that PDMS adsorbs at the surface. However, the formed layer is internally highly mobile and not at all glassy. The size of this layer, which is directly accessed by the time- and length-scale-dependent neutron scattering experiments, significantly exceeds the length scale of the directly adsorbed polymer, presenting the first direct microscopic evidence for the hypothetical interphase.

PDMS with a molecular weight of $17.4 \mathrm{~kg} / \mathrm{mol}$ $\left(M_{w} / M_{n}=1.21\right.$, protonated h-PDMS $)$ and $21.2 \mathrm{~kg} / \mathrm{mol}$ $\left(M_{w} / M_{n}=1.16\right.$, deuterated d-PDMS $)$ was synthesized by living anionic polymerization [15]. With an entanglement molecular weight $M_{e}=12 \mathrm{~kg} / \mathrm{mol}$, these molecular weights in the bulk practically represent an unentangled system. Calculations using density functional theory showed a similar adsorption interaction between the AAO surface and PEO in one case and between the AAO surface and PDMS in another case.

The nanoporous AAO templates (pore diameter $26 \mathrm{~nm}$ ) were prepared by a two-step anodization process [16]. Infiltration of PDMS was performed at room temperature at nitrogen atmosphere during a period of 3 days (see details in the Supplemental Material [17]). The composition of the 
infiltrated polymer mixture (6\% of h-PDMS and $94 \%$ of d-PDMS) was derived from a matching experiment performed with deuterated or protonated dioxane. Compared to the size of the polymer chain (end-to-end distance $R_{e e}=$ $8.5 \mathrm{~nm}$ ), the pore diameter is greater by a factor of 3 . The total amount of polymer infiltrated in 15 AAO templates was approximately $350 \mathrm{mg}$.

The small angle neutron scattering (SANS) experiments were carried out at instrument KWS-2 of the Jülich Centre for Neutron Science (JCNS) at the FRM II reactor in Garching (see Supplemental Material [17]). The PDMS dynamics was investigated using the neutron spin echo (NSE) spectrometer SNS-NSE at the JCNS outstation at the spallation neutron source in the Oak Ridge National Laboratory, USA, and at the J-NSE spectrometer of the JCNS in Garching, Germany. The measurements were performed at $100^{\circ} \mathrm{C}$ in a momentum transfer range $0.12<$ $Q<0.27 \AA^{-1}$ and covered a time range from 1.2 to 160 ns.

The main purpose of the SANS experiment was to control the residual scattering caused by inhomogeneities in the pore filling and/or nonideal matching. From this experiment, we concluded that if the sample is tilted by $25^{\circ}$ relative to the incident beam, in the NSE $Q$ window the total scattering is strongly dominated by the scattering from the polymer (see Supplemental Material [17]).

NSE spectroscopy is a powerful method to study polymer dynamics simultaneously on relevant time and length scales [18]. By using a mixture of protonated and deuterated chains, NSE directly measures the coherent single chain dynamic structure factor $S_{\text {chain }}(Q, t)$ that is the spatial Fourier transform of the monomer-monomer dynamic pair correlation function. Data for two different $Q$ values obtained for the bulk melt and confined PDMS measured at the SNS-NSE spectrometer are presented in Fig. 1. They show a significant slowing down for the confined polymer.

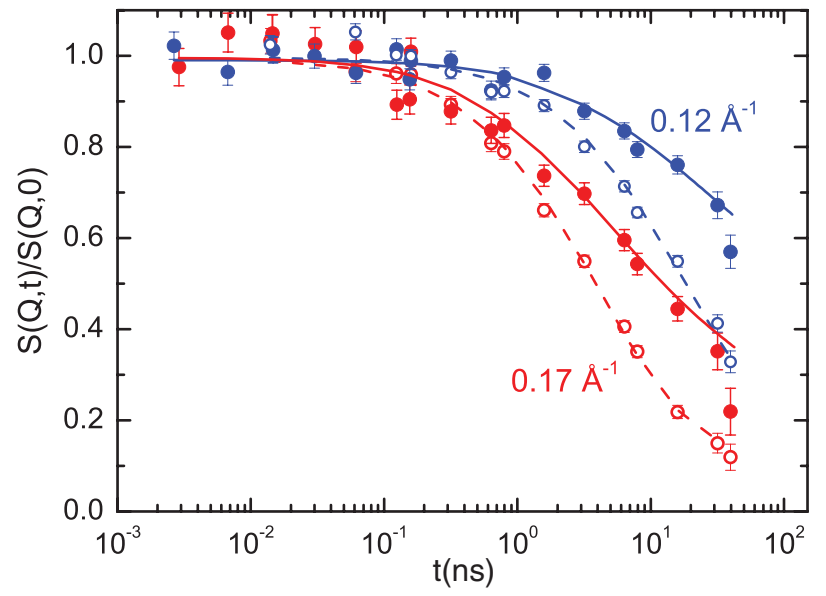

FIG. 1 (color). SNS-NSE data for bulk PDMS (open symbols) and PDMS confined in AAO nanopores (filled symbols). Dashed lines present the Rouse curves with the Rouse rate taken from Ref. [20]. Solid lines represent the final result (see text).
To clarify the origin of the observed slowing we performed an additional experiment at the J-NSE spectrometer that extended the time scale up to 160 ns. In Fig. 2, the data for some values of the momentum transfer measured at both instruments are presented. The results are in a very good agreement. The data display an initial fast decay of the pair correlation function that crosses over into a much weaker decay at long times.

This plateaulike behavior in the single chain dynamic structure factor indicates the presence of a nondecaying part in the pair correlation function that would be caused by an immobilized polymer layer in the vicinity of the pore surface (Fig. 2). In the literature, such immobilized layers near a confining surface were considered glassy. This was concluded from a change of the glass transition temperature but also from "solid-state"-like lines in the NMR spectra, e.g., from PDMS filled with silica nanoparticles [13]. In the context of PEO, the effect was claimed to be independent of temperature, indicating extremely strong adsorption of the polymer segments [7]. However, since the long-time plateau in $S_{\text {chain }}(Q, t)$ significantly depends on the momentum transfer $Q$, such an interpretation does not concur with our results - an immobilized glassy surface layer would lead to a $Q$-independent constant fraction in the structure factor.

Now we quantify our observations: in a first approximation, we describe the long-time tails in $S_{\text {chain }}(Q, t)$ by a $Q$-dependent plateau $\alpha(Q)$. Thus, we have

$$
S_{\text {chain }}(Q, t)=[1-\alpha(Q)] S_{\text {Rouse }}(Q, t)+\alpha(Q),
$$

where $S_{\text {Rouse }}(Q, t)$ is the dynamic structure factor of the Rouse model defined by the formula (see Ref. [19])

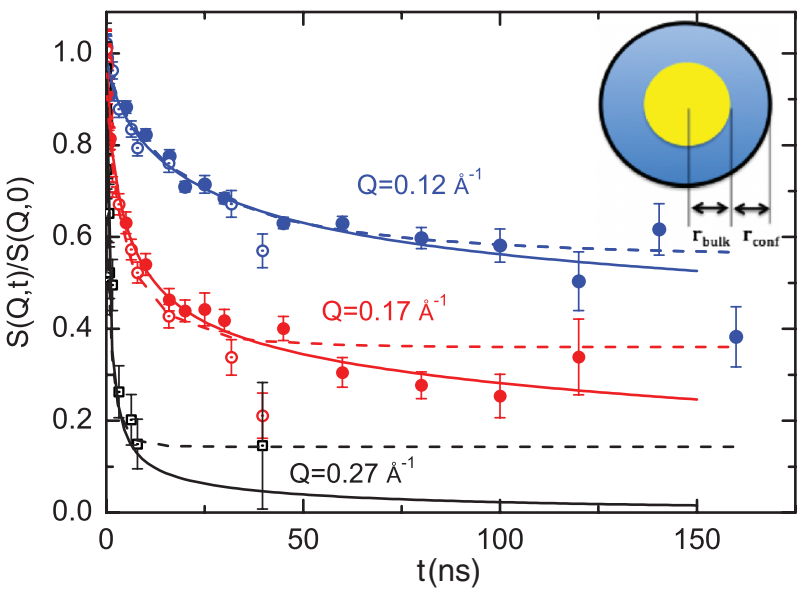

FIG. 2 (color). J-NSE (filled symbols) and SNS-NSE (open symbols) results for confined PDMS. Dashed lines show the fitting of the data using Eqs. (2) and (3). Solid lines present the fitting using a continuous transition region from suppressed to free Rouse modes (see text). A sketch of the two-phase model is shown in the inset. 


$$
\begin{aligned}
S_{\text {Rouse }}(Q, t)= & \frac{1}{N} \exp \left(-Q^{2} D_{R} t\right) \sum_{m, n} \exp \left(-\frac{1}{6}|n-m| Q^{2} l^{2}\right) \\
& \times \exp \left(-\frac{2}{3} \frac{R_{e e}^{2} Q^{2}}{\pi^{2}} \sum_{p=p_{\min }}^{N} \frac{1}{p^{2}}\left\{\cos \left(\frac{p \pi m}{N}\right)\right.\right. \\
& \left.\left.\times \cos \left(\frac{p \pi n}{N}\right)\left[1-\exp \left(-\frac{t p^{2}}{\tau_{R}}\right)\right]\right\}\right)
\end{aligned}
$$

where $p$ counts the Rouse relaxation modes with a wavelength $\mathrm{Nl} / \mathrm{p}$ and $p_{\min }=1 . D_{R}$ is the overall diffusion coefficient, $l$ is the segmental length, and $N$ is the number of segments in the chain. In the fitting, we kept the Rouse time $\tau_{R}$ of bulk PDMS [20] and only varied $\alpha(Q)$. The results are presented by the dashed lines in Fig. 2. The values of $\alpha(Q)$ are given in Table I. As already seen qualitatively, the plateau $\alpha(Q)$ strongly depends on the momentum transfer $Q$.

The presence of a $Q$-dependent plateau in the coherent structure factor of a polymer melt is often associated with an effective confinement length, such as in the reptation tube model of de Gennes. In this model, the plateaus are related to the confinement length (tube diameter $d$ ) by

$$
\alpha(Q) \propto \exp \left(-\frac{Q^{2} d^{2}}{36}\right)
$$

Thus, we can estimate a characteristic confinement length $d$ from the $Q$-dependent plateau. The confinement lengths determined in this way are presented in Table I. The values found are between 3.1 and $3.9 \mathrm{~nm}$, significantly smaller than the tube size in bulk PDMS (approximately $8 \mathrm{~nm}$ [21]). However, the fact that the characteristic confinement length is again $Q$ dependent indicates that a description of the chain dynamics by means of topologically confined chains is not valid.

Summarizing the observations, we can conclude that (i) the dynamics of polymer chains in the pores is significantly slowed compared to that of chains in the bulk; (ii) the concept of two fractions of chains, where one represents a glassy layer and the other displays free Rouse motion is not applicable ( $Q$-dependent plateaus); and finally (iii), the simple approach of a confinement length following the tube model for long entangled chains must obviously be discarded ( $Q$-dependent tube diameter).

TABLE I. Plateau values $\alpha(Q)$ obtained from the fitting of the experimental data using Eq. (1); effective confinement length $d$ estimated using Eq. (3) for given values of the momentum transfer $Q$.

\begin{tabular}{lcc}
\hline \hline$Q(\AA)$ & $\alpha(Q)$ & $d(\mathrm{~nm})$ \\
\hline 0.12 & 0.56 & 3.9 \\
0.17 & 0.36 & 3.6 \\
0.27 & 0.14 & 3.1 \\
\hline \hline
\end{tabular}

From these conclusions, a new model can be derived. This model should contain a fraction of free bulklike chains. Many chains are far from the surface and are not expected to be affected in any way. The second fraction of chains that is close to the surface, however, is assumed to be effectively confined. The dynamic structure factor is presented by the formula

$$
S(Q, t)=A_{\text {bulk }} S_{\text {bulk }}(Q, t)+A_{\text {conf }} S_{\text {conf }}(Q, t),
$$

where $A_{\text {bulk }}$ and $A_{\text {conf }}=1-A_{\text {bulk }}$ are the fractions of the bulk and confined phases respectively; $S_{\text {bulk }}(Q, t)$ is the dynamic structure factor of the free chain defined by Eq. (2), and $S_{\text {conf }}(Q, t)$ is the dynamical structure factor of the confined chain that is modeled by a modified Rouse ansatz [Eq. (2) with $p_{\min }>1$ ]. The effective confinement is taken into account by suppressing the center-of-mass diffusion and the first modes of $p<p_{\min }$ with wavelengths longer than the distance between chain adsorption points [22].

This model contains only two variable parameters: the fractions of bulk $A_{\text {bulk }}$ and confined $A_{\text {conf }}=1-A_{\text {bulk }}$ polymer and the number of suppressed Rouse modes $p_{\text {supp }}=$ $p_{\text {min }}-1$, reflecting the effective confinement length. By the fitting of all $Q$ simultaneously, the amount of the bulk phase was found to be $30 \%$ of the total amount of polymer in the pores. The value of $p_{\text {supp }}$ was found to be 4 . The longest mode that still fully contributes to the relaxation $p_{\min }=5$ has a wavelength corresponding to 46 monomers that are taken as segments. The end-to-end distance of a subchain corresponding to 46 segments gives an effective confinement length $d=R_{e e}=\sqrt{N l^{2}}=3.8 \mathrm{~nm}$. The quality of the data description (not shown) is very similar to that of Eqs. (1) and (2) (dashed lines in Fig. 2). However, now the plateau levels find a natural explanation.

Using the concept of suppressed Rouse modes for the description of the confined phase has limitations. They are related to the different possible conformations of the

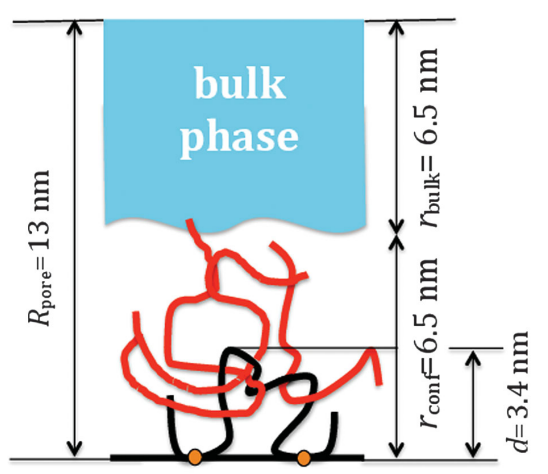

FIG. 3 (color). Schematic representation of the artificial surface-induced entanglements in the confined polymer melt. The black line represents the chain adsorbed on the surface of an AAO nanopore, and the red lines show entangled chains in the confined phase. 
polymer chain in the confined phase. In particular, differently anchored chains can have a different number of segments between the anchor points and/or contain free ends. This results in different values for the number of suppressed modes $p_{\text {supp }}$ for different chains in the confined melt.

In order to account for the variety of anchored conditions, we introduced a smoothly varying cutoff function (modeled by a Fermi function) that increases the mode relaxation rate gradually from virtually zero to the standard Rouse model rate as a function of $p$. Whereas in the discrete approach the transition from existing to suppressed modes was sharp, with an additional parameter $\Delta p_{\text {supp }}$, a width of this transition region is introduced. The improvement of the quality of the fitting is remarkable (full lines in Fig. 2) resulting in $p_{\min } \approx 6, \Delta p_{\text {supp }}=1.1$ and $25 \%$ of bulk and $75 \%$ of confined polymer. The mode $p_{\text {min }} \approx 6$ has a wavelength corresponding to 38 segments. The end-to-end distance of a subchain corresponding to 38 segments gives an effective confinement length $d=3.4 \mathrm{~nm}$.

The obtained distribution of bulk and confined chains can be transferred to a corresponding layer thickness. We find a layer of confined chains with a thickness $r_{\text {conf }}=$ $6.5 \mathrm{~nm}$ and a cylindrical bulk phase again with a radius of $r_{\text {bulk }}=6.5 \mathrm{~nm}$ (see inset in Fig. 2). The fact that the size of a PDMS subchain between the anchored points is $3.4 \mathrm{~nm}$ clearly indicates that the layer with a thickness of $6.5 \mathrm{~nm}$ is not only built by chains adsorbed at the surface but rather supports a situation as illustrated in Fig. 3. The polymer chains anchored to the surface may form loops; neighboring chains then can interpenetrate these loops. These may only diffuse by reptation. Estimating the reptation time based on the confinement length of $3.4 \mathrm{~nm}$ yields a reptation time that is 60 times longer than maximum Fourier time. It follows that neither the anchored nor the entangled chains exhibit center-of-mass diffusion in the time frame of our experiment. These penetrating chains can be considered as the interphase (transitional phase) between the polymer adsorbed on the surface and the bulk polymer phase. This interphase is formed merely as a consequence of confinement and dramatically changes the properties compared to those of the unentangled polymer melt. To our knowledge, our observation constitutes the first direct and quantitative observation of the often invoked interphase on a microscopic scale. To summarize, the study of an unentangled polymer melt confined in cylindrical nanopores was performed using NSE spectroscopy. The dynamic behavior of PDMS is strongly affected by the confinement and follows a two-phase model: one free bulklike fraction of chains and one phase of confined polymers. This phase is characterized by a vanishing center-of-mass diffusion and by a suppression of long wavelength Rouse modes as expected for a multiple anchored and topologically confined chain. By fixing the segmental friction to the value of the bulk polymer, a very good description of the data was achieved. The resulting fitting parameters yield a $75 \%$ fraction of confined PDMS chains and a $25 \%$ fraction of bulklike chains displaying free Rouse behavior. The corresponding confined layer of $6.5 \mathrm{~nm}$ thickness (Fig. 3) on one hand consists of a dominant fraction of highly mobile segments and on the other hand evidences the presence of a polymer interphase induced by the interaction of the anchored polymer and the surrounding melt.

The support of the DFG Priority Program SPP1369 Polymer-Solid Contacts: Interfaces and Interphases is gratefully acknowledged. We thank F.-J. Köhne (FZJ/ PGI-6) for help with the sample preparation. A. A. and J.C. acknowledge financial support from Projects No. MAT2007-63681 and No. IT-436-07 (G. V.).

[1] R. Zorn, L. v. Eijck, M. M. Koza, and B. Frick, Eur. Phys. J.: Spec. Top. 189, 1 (2010).

[2] R. Berwanger, A. Henschel, K. Knorr, P. Huber, and R. Pelster, Phys. Rev. B 79, 125442 (2009).

[3] F. Barroso-Bujans, F. Fernandez-Alonso, J. A. Pomposo, S. Cerveny, A. Alegra, and J. Colmenero, ACS Macro Lett. 1, 550 (2012).

[4] A. Starzyk and M. Cieplak, J. Chem. Phys. 135, 235103 (2011).

[5] M. Muthukumar, Adv. Chem. Phys. 149, 129 (2012).

[6] P. J. Dionne, R. Ozisik, and C. R. Picu, Macromolecules 38, 9351 (2005).

[7] S. Y. Kim, H.W. Meyer, K. Saalwächter, and C. F. Zukoski, Macromolecules 45, 4225 (2012).

[8] J. Martín, M. Krutyeva, M. Monkenbusch, A. Arbe, J. Allgaier, A. Radulescu, P. Falus, J. Maiz, C. Mijangos, J. Colmenero, and D. Richter, Phys. Rev. Lett. 104, 197801 (2010).

[9] K. Nusser, G. J. Schneider, and D. Richter, Soft Matter 7, 7988 (2011).

[10] A. Papon, H. Montes, M. Hanafi, F. Lequeux, L. Guy, and K. Saalwächter, Phys. Rev. Lett. 108, 065702 (2012).

[11] T. V.M. Ndoro, M.C. Böhm, and F. Müller-Plathe, Macromolecules 45, 171 (2012).

[12] D. Ciprari, K. Jacob, and R. Tannenbaum, Macromolecules 39, 6565 (2006).

[13] A. Şerbescu and K. Saalwächter, Polymer 50, 5434 (2009).

[14] S. Bhattacharya, A. Milchev, V. G. Rostiashvili, A. Y. Grosberg, and T.A. Vilgis, Phys. Rev. E 77, 061603 (2008).

[15] V. Bellas, H. Iatrou, and N. Hadjichristidis, Macromolecules 33, 6993 (2000).

[16] H. Masuda and K. Fukuda, Science 268, 1466 (1995).

[17] See the Supplemental Material at http://link.aps.org/ supplemental/10.1103/PhysRevLett.110.108303 for a detailed description of the SANS experiment.

[18] D. Richter, M. Monkenbusch, A. Arbe, and J. Colmenero, in Neutron Spin Echo in Polymer Systems, Advances in Polymer Science Vol. 174 (Springer, Berlin, 2005), p. 1. 
[19] M. Doi and S.F. Edwards, The Theory of Polymer Dynamics (Clarendon Press, Oxford, 1986).

[20] D. Richter, B. Ewen, B. Farago, and T. Wagner, Phys. Rev. Lett. 62, 2140 (1989).
[21] F. J. Fetters, D. J. Lohse, D. Richter, T. Witten, and A. Zirkel, Macromolecules 27, 4639 (1994).

[22] D. Richter, L. Willner, A. Zirkel, B. Farago, L. J. Fetters, and J. S. Huang, Phys. Rev. Lett. 71, 4158 (1993). 